\title{
ERRATUM
}

\section{Lenalidomide inhibits osteoclastogenesis, survival factors and bone-remodeling markers in multiple myeloma}

I Breitkreutz, MS Raab, S Vallet, T Hideshima, N Raje, C Mitsiades, D Chauhan, Y Okawa, NC Munshi, PG Richardson and $\mathrm{KC}$ Anderson

Leukemia (2008) 22, 1973; doi:10.1038/leu.2008.216

Correction to: Leukemia (2008) 22, 1925-1932;

doi:10.1038/leu.2008.174; published online 3 July 2008

Owing to a typesetting error, Figure $2 b$ of the above article was published incorrectly.
The correct figure is reproduced here.

The publisher apologizes for this error and any inconvenience it may have caused.
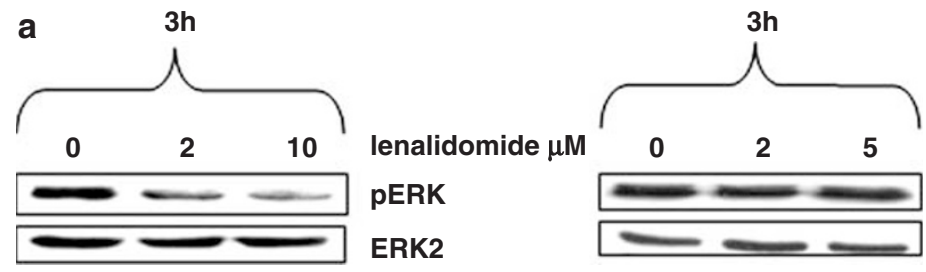

bortezomib nM

PERK

ERK2

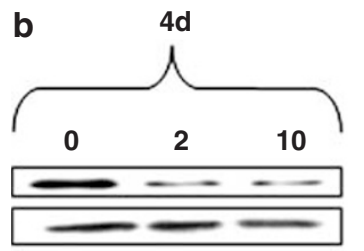

lenalidomide $\mu \mathrm{M}$

PU.1

Actin

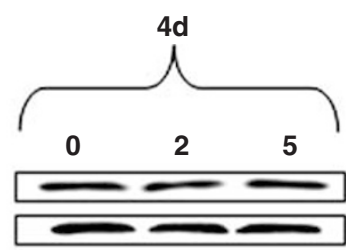

bortezomib nM

PU.1

Actin
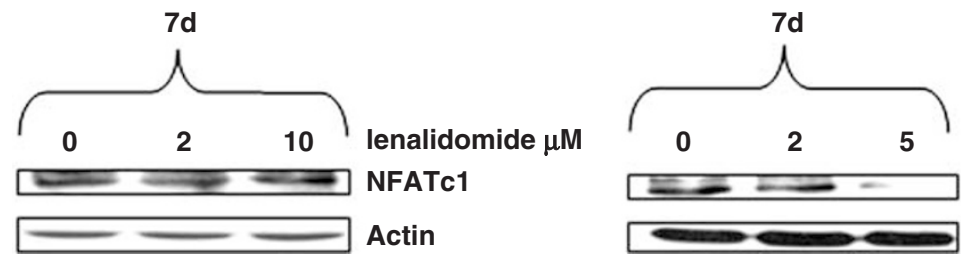

bortezomib nM

NFATc1

Actin

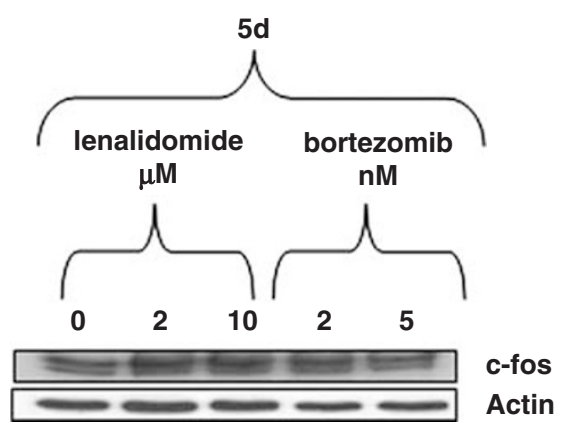

Figure 2 Effect of lenalidomide and bortezomib on transcription factors during osteoclastogenesis. (a) Lenalidomide $(100 \%$ at $0 \mu \mathrm{M}, 60.2 \%$ at $2 \mu \mathrm{M}$ and $48.8 \%$ at $10 \mu \mathrm{M})$, but not bortezomib, resulted in a dose-dependent inhibition of extracellular signal-regulated kinase (ERK) phosphorylation in peripheral blood mononuclear cells (PBMCs) incubated with macrophage colony-stimulating factor (M-CSF) and receptor activator of NF- $\mathrm{KB}$ ligand (RANKL) for $3 \mathrm{~h}$. (b) Lenalidomide, but not bortezomib, resulted in a decrease of PU.1 after incubation of PBMCs for 4 days in the presence of RANKL and M-CSF. Conversely, bortezomib, but not lenalidomide, downregulated NFATc1. C-fos was not downregulated by either agent at 5 days. 